\title{
Clinical and radiological evaluation of furcal perforation repaired by mineral trioxide aggregate and intermediate restorative material
}

\author{
Kamrun Naher Shomi, Mozammal Hossain and Md. Shamsul Alam
}

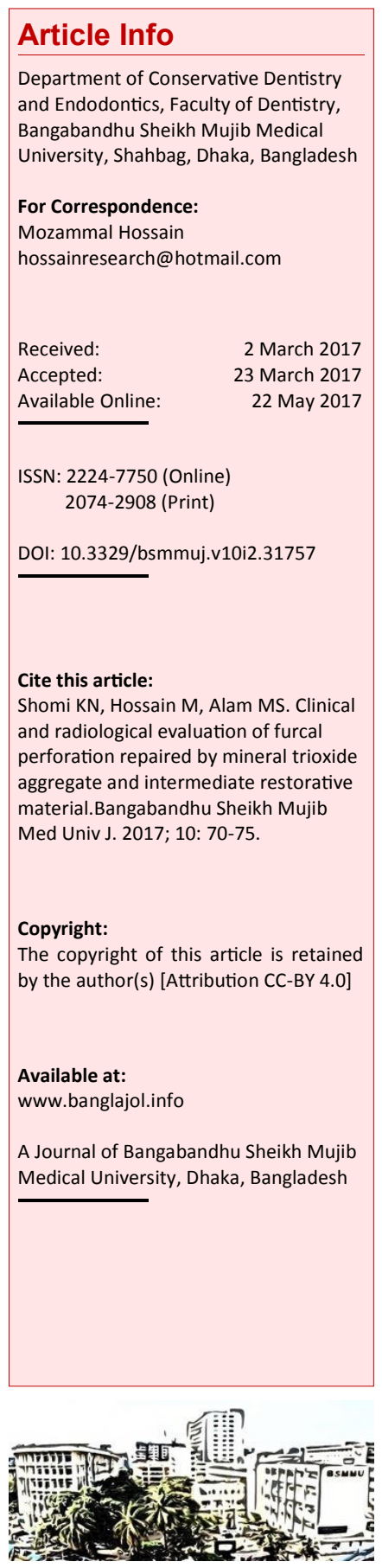

\section{Abstract}

The purpose of the present study was to assess the clinical and radiological outcome following repair of furcal perforation by mineral trioxide aggregate (MTA) and intermediate restorative material (IRM) in mandibular molar teeth. Forty teeth having furcal perforation were enrolled in this study, out of which 20 teeth were treated with MTA and the remaining 20 teeth were subjected to IRM treatment. Following perforation repair, all teeth were subjected to root canal treatment followed by final restoration. Clinical and radiological outcome was evaluated at 3, 6 and 12 months interval. The results showed that in both MTA and IRM groups, pain, tenderness on percussion as well as swelling and sinus was gradually decreased with the increase of the observation period. Furthermore, the widening of the periodontal ligament space and communication with the oral cavity were gradually decreased. Although there was no significant differences between MTA and IRM at 3 and 6 months observation period but at 12 months, the clinical outcome between MTA and IRM was statistically significant $(p<0.05)$. It can be concluded that repair of furcal perforation by MTA showed more effective than that of IRM.

\section{Introduction}

Furcal perforation is considered as one of the major complications leading to the failure of endodontic treatment. 1 It usually occurs during the preparation of an endodontic access cavities or exploring canal orifice of multirooted teeth.? Therefore, it is defined as an artificial opening in a tooth or its root created by boring, piercing cutting or pathologic resorption which results in a communication between the pulp space and the periodontal tissue. This artificial communication results in the irreversible loss of periodontal attachment in the area as well as surrounding periodontium at the perforation site due to mechanical trauma and to the introduction of microbially derived substances that inevitably accompany the perforation. $\underline{3}$

A variety of materials have been applied for the repair of furcal perforation that includes amalgum, intermediate restorative material (IRM), guttapercha, dentine chips, calcium hydroxide, cavit, tricalcium phosphate, hydroxyapatite, glass ionomer cement, super EBA. .4 However, none of the above materials have showed adequate repair of this iatrogenic accident. $\frac{5}{5}$ Therefore, interest has centered on the use of mineral trioxide aggregate (MTA), which is considered to fulfill the requirement of an ideal material for perforation repair. $\underline{5,6}$
MTA is a mineral powder consisting of hydrophilic particles whose principal components are tricalcium silicate, tricalcium aluminate, tricalcium oxide and other mineral oxides. 7 It has a $\mathrm{pH}$ of 12.5 and sets in the presence of moisture in approximately 4 hours. 7 It is an ideal material for the perforation repair due to its favorable properties that includes good sealing capability, biocompatibility, bactericidal activity, radiopacity, and the ability to set in the presence of blood or tissue fluids. .8 Moreover, several in vitro studies have demonstrated that it has excellent sealing ability and biocompatibility when used different leakage approaches, fluid filtration technique, dye-leakage model, bacterial leakage model, and dye-excretion leakage method. 8 It is also capable to protect the pulp when used as pulp capping agent, repair of the periodontium and new cementum formation over the material, and promote the growth of the cementum and formation of the bone. $\frac{8,9}{9}$ MTA experimentally showed better sealing ability than that of other materials such as amalgam, zinc oxide eugenol cement, resin modified cement and IRM.9-12 Therefore, it is considered that MTA may also be suitable for closing the communication between the pulp chamber and the underlying periodontal tissues. 5 In the present study, the clinical and radiological outcome following repair of the furcal perforation using MTA was assessed and 
compared the results with that of IRM.

\section{Materials and Methods}

This study was performed during the period from May 2014 to April 2015. Forty patients referred with the complaint of iatrogenic perforation in the furcal area of the mandibular molar teeth (age range of the patient was 18 to 45 years old). Teeth were divided by simple random sampling (by lottery methods) into two groups as follows: In Group I, 20 teeth with furcal perforation were repaired by MTA and in Group II, 20 teeth were treated by IRM. A written informed consent was taken from the patients or patient's guardian. Patient's symptoms, clinical sings, detailed medical and dental history were recorded. A preoperative radiograph was taken in each case. The radiograph was examined by the same dental radiologist and recorded on the date collection sheet.

\section{Repair of furcal perforation}

Disinfection of the operative field and proper sterilization of instruments were done in every case in an aseptic manner. A preoperative intraoral radiograph was taken and then infiltration of local anesthesia was given. Isolation of teeth was done with cotton roll and saliva ejector. Temporary restoration was removed and coronal access opening was modified and then the canal orifice was located followed by negotiation of the canal; a \# $10 \mathrm{k}$ file was inserted in to the bleeding point and an intra oral periapical radiograph was obtained. Working length was determined by radiograph. The root canals were irrigated with $5.2 \%$ sodium hypochlorite and normal saline and enlarge up to $25 \mathrm{k}$ file at the measured working lengths. The canals were blocked with GP before perforation repair so that MTA or IRM could not flow to the canal orifice. Bleeding from the perforation site was controlled with firm pressure by moistened cotton pellet.

In Group I, MTA powder was mixed on a paper pad with distilled water 3:1 powder water ratio. They were mixed for about $1 \mathrm{~min}$ to ensure all powder particles are hydrated by gain a creamy consistency. Once the material acquired this consistency, it was applied with the help of amalgum carrier and gently packed into the perforation site with hand plugger and pressed with moistened cotton pellet. The GP were removed from the root canal the access cavity was sealed with cotton pellet followed by a layer of $\mathrm{ZnO}$ eugenol cement. At second visit, access cavity was reopened. The root canals were then obturated with gutta percha point and sealer using the lateral condensation technique. Access was sealed with GI and composite resin restoration. A post operative radiograph was taken.
In Group II, IRM was mixed according to manufacturer instruction and applied over the perforation area with the help of the amalgam carrier and condensed with the hand plugger. The root canal was then obturated with gutta perch point and sealer using the lateral condensation technique. The postoperative radiograph was taken, cheeked and adjusted the occlusion as needed.

\section{Clinical and radiological evaluations}

The patients were recalled for clinical and radiographic evaluations after 3,6 , and 12 months interval. Clinical and radiological outcome were assessed for each patient by the designated investigators at every follow-up visit by the presence or absence of signs and symptoms. Blind to the treatment record, two evaluators assessed the clinical and radiological findings, as follows: a) Pain was assessed according to VAS (Visual analogue scale) system. In this method, VAS of a $10 \mathrm{~cm}$ long horizontal line with points labeled as $(0)$ no pain, (13) mild pain, (4-6) moderate pain, (7-10) severe pain. In data collection sheet pain recorded as, $0=$ no pain, $1=$ mild pain, 2 = moderate pain, $3=$ severe pain. b) The tenderness on percussion of tooth was performed by gentle tapping with the blunt handle of mouth mirror on the offending tooth. Degree of response to percussion is directly proportional to degree of inflammation. c) Evidence of swelling was observed by carefully palpate the swelling to ascertain it's tissue of origin. d) Evidence of sinus tract was observed by visual inspection of the soft tissues was made for any sinus tract of endodontic origin. It was assessed by inserting \# 30 number gutta-percha through elevated nodule of tissue and examined in radiograph. e) Radiographic assessment was performed blind by the same dental radiologist in using a magnifier. The periodontal ligament space after perforation repair and communication between the perforation and oral cavity were assessed.

\section{Statistical analysis}

Statistical analysis of the results were done by using computer based statistical softer, SPSS 20.00 version (SPSS Inc. Chicago, USA). Significant of difference between two groups were performed by Chi-square test and a value of $\mathrm{p}<0.05$ was considered as statistically significant.

\section{Results}

The results of pain showed that 17 of MTA treated teeth, patient did not complained of pain at 3 months, followed by 18 at 6 months and 19 at 12 months observation period. On the other hand, at 3 , 6 and 12 months observation period, the results of pain in IRM treated teeth were 11, 12, 14, respectively. The results of pain between two groups were statistically significant $(\mathrm{p}<0.05)$ at 12 
months (Table I). However, 3 MTA treated teeth had mild pain at 3 months, followed by 2 at 6 months, and one at 12 months observation period. On the other hand, 9 cases of IRM treated teeth had mild pain at 3 months followed by 8 at 6 months and 6 at 12 months.

Regarding tenderness on percussion, the results showed that tenderness was gradually decreased in both MTA and IRM. It was found that 11 MTA treated teeth had tenderness at 3 months, followed by 4 at 6 months and 1 at 12 months observation period. On the other hand, 17, 12, 6 of IRM treated teeth had pain at 3, 6 and 12 months, respectively. The results between two groups were statistically significant $(p<0.05)$ at 12 months (Table I).

The results of swelling showed that at 3 months, no case of swelling was found in MTA treated teeth but 4 cases of IRM showed swelling. At 6 and 12 months observation period, 1 MTA and 6 IRM had swelling. Furthermore, the results of sinus tract showed that at 3 months, no case of MTA treated teeth had sinus tract but 4 cases of IRM had sinus tract. At 6 and 12 months observation period, 1 MTA and 6 IRM had sinus tract. The results were

\section{Table I}

Clinical and radiological results at 12 months

\begin{tabular}{|c|c|c|c|c|}
\hline Clinical evaluation & Criteria & $\begin{array}{l}\text { Group-I } \\
\text { (MTA) } \\
(\mathrm{n}=20)\end{array}$ & $\begin{array}{l}\text { Group-II } \\
\text { (IRM) } \\
(\mathrm{n}=20)\end{array}$ & $\begin{array}{c}\mathrm{p} \\
\text { value }\end{array}$ \\
\hline \multirow[t]{4}{*}{ Pain by VAS } & No pain & 19 & 14 & \multirow{4}{*}{0.037} \\
\hline & Mild pain & 1 & 6 & \\
\hline & $\begin{array}{l}\text { Moderate } \\
\text { pain }\end{array}$ & 0 & 0 & \\
\hline & $\begin{array}{l}\text { Severe } \\
\text { pain }\end{array}$ & 0 & 0 & \\
\hline \multirow[t]{2}{*}{ Pain on percussion } & Present & 1 & 6 & \multirow[t]{2}{*}{0.037} \\
\hline & Absent & 19 & 14 & \\
\hline \multirow[t]{2}{*}{ Swelling } & Present & 1 & 6 & \multirow[b]{2}{*}{0.037} \\
\hline & Absent & 19 & 14 & \\
\hline \multirow[t]{2}{*}{ Sinus tract } & Present & 1 & 6 & \multirow{2}{*}{0.037} \\
\hline & Absent & 19 & 14 & \\
\hline \multirow[t]{2}{*}{ Peridontal widening } & Present & 18 & 9 & \multirow{2}{*}{0.002} \\
\hline & Absent & 2 & 11 & \\
\hline \multirow{2}{*}{$\begin{array}{l}\text { Communication between } \\
\text { the perforation and oral } \\
\text { cavity }\end{array}$} & Present & 19 & 14 & \multirow{2}{*}{0.037} \\
\hline & Absent & 1 & 6 & \\
\hline \multirow[t]{2}{*}{ Final outcome } & Present & 19 & 14 & \multirow{2}{*}{0.037} \\
\hline & Absent & 1 & 6 & \\
\hline
\end{tabular}

Data were expressed in number; Statistical analysis was done by Chi-square test was performed to compare between groups; $\mathrm{n}=$ Number of subject statistically significant $(\mathrm{p}<0.05)$ at 12 months.

Radiological evaluations showed that periodontal ligament space was gradually decreased following MTA and IRM therapy. At 3 months, in 9 MTA treated teeth, there were a decreased in the widening of periodontal ligament space, followed by 16 at 6 months and 18 at 12 months. On the other hand, 3, 7 and 9 cases of IRM had a decrease in the widening of periodontal ligament space at 3, 6 and 12 months. The differences between MTA and IRM were statistically significant at 12 months. Furthermore, communication between the perforation and oral cavity was found in 2 cases of MTA and 8 cases of IRM at 3 months, followed by 1 cases of MTA and 6 cases of IRM at 6 and 12 months, respectively. The remaining teeth successfully repaired the communication between the perforation and oral cavity. The differences between MTA and IRM was statistically significant $(p<0.05)$ at 12 months (Figure 1, Figure 2). Furthermore, the final outcome of MTA and IRM was 95 and 75\%, respectively, which was statistically significant.

\section{Discussion}

In the present study, perforation was sealed by MTA or IRM and the clinical and radiographical investigations were performed. Treatment usually consists of removal of temporary restoration, modification of coronal access opening, determination of working length, control of bleeding at the perforation site, and then the canals were dried followed by sealed the perforation with either by MTA or IRM. After perforation repair, all teeth were subjected to root canal treatment followed by final restoration. The procedure used in the present study is originally based on previous studies. $\underline{13,14}$

In the present study, it was found that pain gradually decreased following MTA therapy. It was found that 17 cases of MTA treated teeth had no pain at 3 months, followed by 18 cases at 6 months and 19 at 12 months observation period. Previous studies have reported that MTA can reduce pain due to its high $\mathrm{pH}$ as well as antibacterial activity, excellent biocompatibility and stimulate mineralization. $\underline{8}$ Furthermore, MTA provides an effective seal of furcal perforations and can be considered a potential material enhancing the prognosis of perforated teeth that can also reduce bacterial penetration. 4 However, 3 cases of MTA treated teeth had mild pain at 3 months, followed by 2 cases at 6 months, and 1 at 12 months observation period. On the other hand, 9 cases of IRM treated teeth had mild pain at 3 months followed by 8 cases at 6 months and 6 cases at 12 months. The reason of pain occurs following treatment either with MTA or IRM is not clearly understood from the present study. However, 


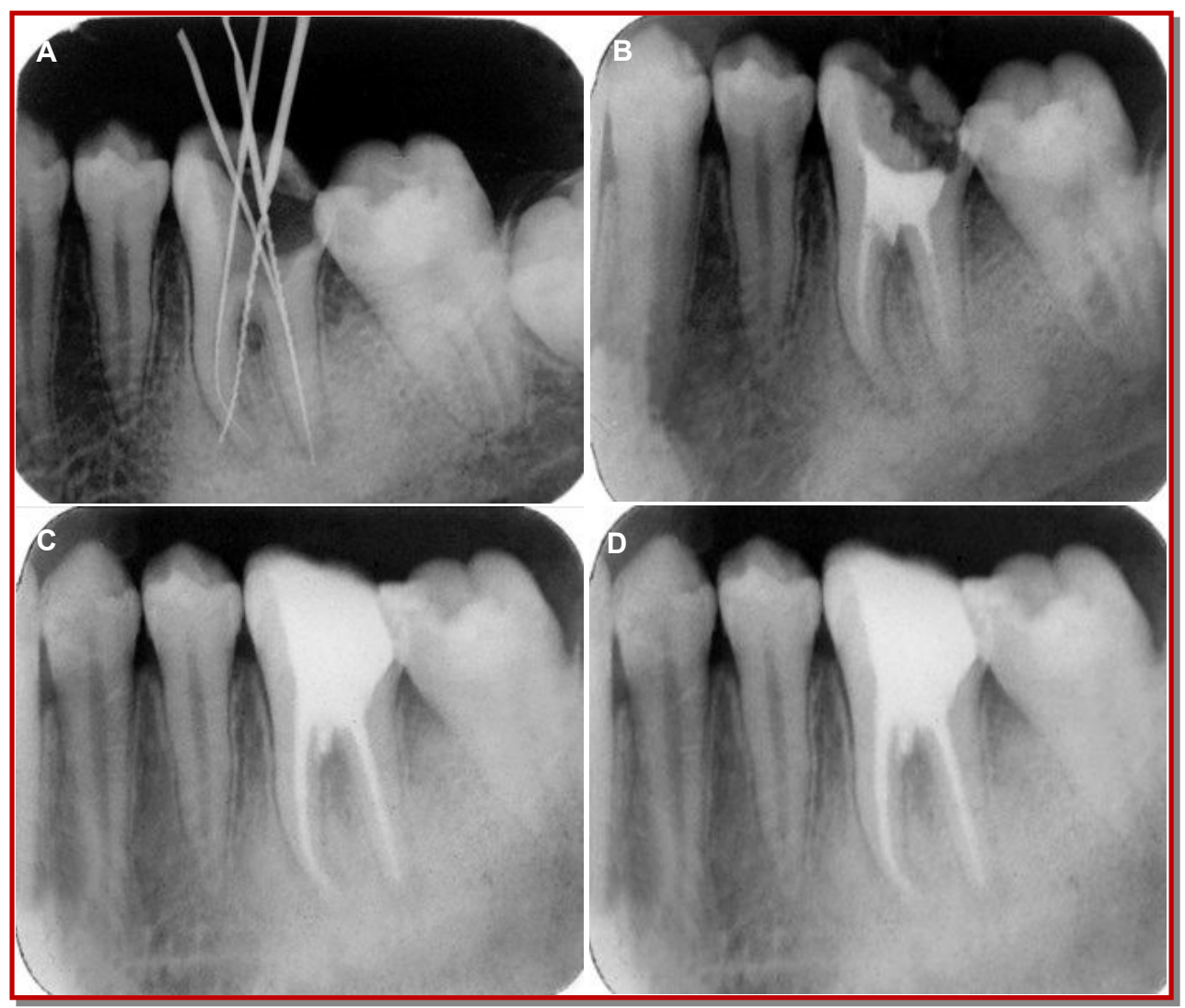

Figure 1: The representative radiological photographs of perforation repair by MTA. Floor perforation (A), Perforation repair by IRM (B), After obturation (C), and at 12 months observation period (D)

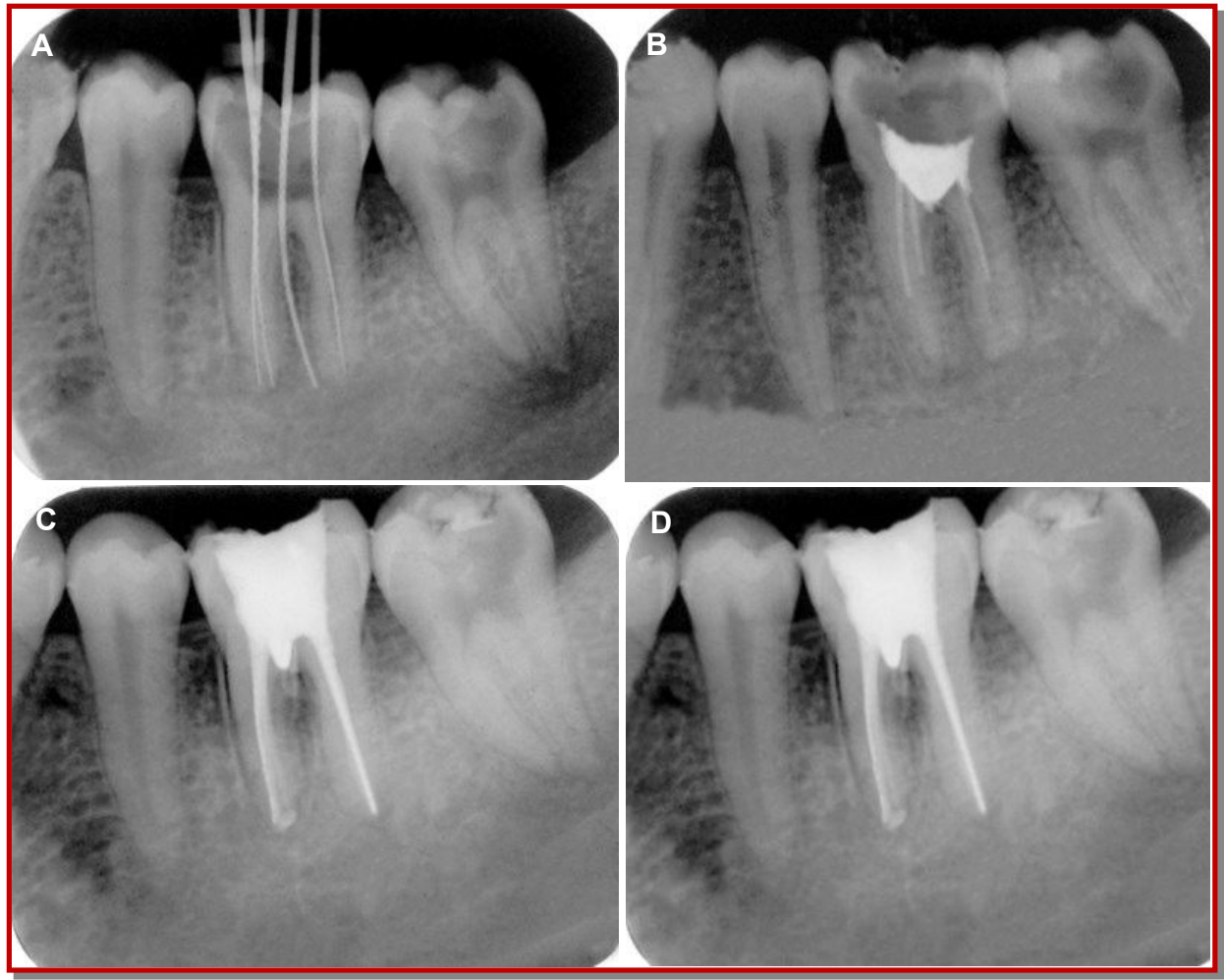

Figure 2: The representative radiological photographs of perforation repair by IRM. Floor perforation (A), Perforation repair by IRM (B), After obturation (C), and At 12 months observation period (D) 
incidence of pain by MTA or IRM may be due inadequacy of the repair, $\underline{15}$ or extrusion of the material into the furcal area.? Furthermore, it is reported that IRM causes persistent inflammation and slower healing potential, $\underline{\underline{16}}$ and it is more cytotoxic than the MTA. 17 It might be the possible reason for higher incidence of pain in IRM treated teeth.

Regarding tenderness on percussion, it was found that tenderness gradually decreased in both MTA and IRM. However, the differences between two groups were statistically significant $(p<0.05)$ at 12 months. Previous studies have indicated that tenderness following MTA therapy may be reduced by its antimicrobial activity due to high $\mathrm{pH}$, and promotes growth of the cementum and formation of bone, which promotes biological repair and regeneration of periodontal ligament.18 On the other hand, as IRM is a polymer reinforced eugenol based material, like eugenol when IRM contact either directly during extrusion/ overfilling or through its diffusible substances, which may leach into periradicular tissues. If this occurs, these destruction and inflammation of periradicular tissues, which manifest as pain, tenderness on percussion.

The results of swelling and sinus tract showed that at 3 months, no cases of MTA had swelling or sinus tract. However, at 6 and 12 months, 1 MTA and 6 IRM had both swelling and sinus tract. In both groups, the presence of sinus tract and swelling might be due to incomplete seal of the furcation defects as reported by a previous study. .5

The results of widening of periodontal ligament space revealed that it gradually decreased following both MTA and IRM therapy. At 3 months, in 9 MTA treated teeth, there were a decreased widening of periodontal ligament space followed by 16 at 6 months and 18 at 12 months. On the other hand, 3 IRM, 7 and 9 cases of IRM showed a decrease in the widening of periodontal ligament space. The differences between MTA and IRM were statistically significant at 12 months. Previous studies have indicated that MTA stimulates interlukin (IL-6 and IL-8) production, allows the overgrowth of cementum (osteo-cementum) and formation of bone, which facilitates the regeneration of periodontal ligament.19 On the other hand, IRM has the capacity to provide satisfactory sealing which might reduce micro-leakage thus decreasing the thickness of periodontal ligament.

The results of communication between the perforation and oral cavity showed that at 3 months communication was found in 2 cases of MTA and 8 cases of IRM, followed by 1 case of MTA and 6 cases of IRM at 6 and 12 months observation periods. The remaining teeth successfully repaired the communication between the perforation and oral cavity. The difference between MTA and IRM was statistically significant $(\mathrm{p}<0.05)$ at 12 months. One previous study indicated that MTA have superior sealing ability than the IRM when used for perforation repair. $\underline{19}$ It also has the capability to promote the hard tissue formation. MTA may actually promote bone turnover by increasing the osteoclastic and osteoblastic activity. On the other hand, there is no evidence of inhibition of dentoalveolar or osseous wound healing associated with IRM.

The final outcome of MTA and IRM was 95\% and $70 \%$, respectively. Several studies were corresponded to the present studies that $100 \%$ success rate was achieved in MTA treated teeth.7,13,14 These studies showed that perforation repair by MTA were successful both in clinical and radiological examinations. The final outcome of MTA of the present study was corresponded to that of previous studies. $.7,13,14$

\section{Conclusion}

MTA is better than IRM in this study as a furcul perforation repair materials in mandibular molar tooth.

\section{Ethical Issue}

The research protocol was approved by the committee and permission for the study was taken from the Institutional Review Board of Bangabandhu Sheikh Mujib Medical University.

\section{Acknowledgement}

This work was supported by the Research Grant for Student, Bangabandhu Sheikh Mujib Medical University.

\section{References}

1. Vanni JR, Della-Bona A, Figueiredo JAPD, Pedro G Voss D, Kopper PMP. Radiographic evaluation of furcal perforations sealed with different materials in dog's teeth. J Appl Oral Sci. 2011; 19: 421-25.

2. Shah D, Manwar NU, Chandak M, Pattanaik N. Repair of furcal perforation with mineral trioxide aggregate: Case report. J Dent Med Sci. 2013; 9: 6669.

3. Castellucci A. The use of mineral trioxide aggregate to repair iatrogenic perforations. Dent Today. 2008: $48-51$.

4. De-Dues G, Petruccelli V, Gurgel-Filho E, Coutinho -Filho T. MTA versus Portland cement as repair material for furcal perforations. Int Endod J. 2006; 
39: 293-98.

5. Arens DE, Torabinejad M. Repair of furcal perforations with mineral trioxide aggregate: Two case reports. Oral Surg Oral Med Oral Pathol Oral Radiol. 1996; 82: 84-88.

6. Pitt Ford TR, Torabinejad M, McKendry DJ, Hong $\mathrm{CU}$, Kariyawasam SP. Use of mineral trioxide aggregate for repair of furcal perforations. Oral Surg Oral Med Oral Pathol Oral Radiol Endod. 1995; 79: 756-63.

7. Unal GC, Maden M, Isidan. Repair of furcal iaterogenic perforation with mineral trioxide aggregate: Two years follow-up of two cases. Eur J Dent. 2010; 4: 475-81.

8. Torabinejad M, Hong CU, Pitt Ford TR, Kettering JD. Cytotoxicity of four root end filling materials. J Endod. 1995; 21: 489.

9. Sultana R, Hossain M, Alam M. Evaluation of clinical and radiological outcomes of mineral trioxide aggregate and calcium hydroxide as indirect pulp capping agents in the treatment of deep carious lesion of permanent teeth. Bangabandhu Sheikh Mujib Med Univ J. 2016; 9: 140-45.

10. Nakata TT, Bae KS, Baumgartner JC. Perforation repair comparing mineral trioxide aggregate and amalgam using an anaerobic bacterial leakage model. J Endod. 1998; 24: 184-86.

11. Lee YC, Yang SF, Hwang YF, Chueh LH, Chung $\mathrm{KH}$. Microleakage of endodontic temporary restorative material. J Endod. 1993; 19: 516-20.
12. Daoudi MF, Saunders WP. In vitro evaluation of furcal repair using mineral trioxide aggregate or resin modified glass ionomer cement with and without the use of the operating microscope. J Endod. 2002; 28: 512-15.

13. Silveria CMM, Sanches-Ayala A, Legravere MO, Pilatti G, Gomes OMM. Repair of furcul perforation with mineral trioxide aggregate: Long-term followup of two cases. J Can Dent Assoc. 2008; 74: 729-33.

14. Bora A, Das PK, Banerjee S, Datta P, Goswami A, Pandey MB, Zahir S, Kundu GK. Repair of laterogenic perforation with mineral trioxide aggregate: A case report. JDMSc. 2015; 14: 73-76.

15. Alhadainy HA. Root perforations. A review of literature. Oral Surg Oral Med Oral Pathol. 1994; 78: 368-74.

16. Meher WP, Johnson RL, Hess J, Steiman HR. Biocompatibility of retrograde filling materials in the ferret canine Amalgam and IRM. Oral Surg Oral Med Oral Pathol. 1992; 73: 738-45.

17. Mozayeni MA, Salem Milani A, Alim Marvasti L, Mashadi Abbas F, Modaresi SJ. Cytotoxicity of cold ceramic compared with MTA and IRM. Iran Endod J. 2009; 4: 106-11.

18. Bogen G, Kuttler S. Mineral trioxide aggregate obturation: A review and case series. J Endod. 2009; 36: 779-90.

19. Clauder T, Shin SJ. Repair of perforations with MTA: Clinical applications and mechanisms of action. Endo Topics. 2006; 15: 32-55. 